\title{
BREAKTHROUGH RENEWABLES AND THE GREEN PARADOX
}

\author{
Frederick van der Ploeg, University of Oxford ${ }^{*}$
}

\begin{abstract}
We show how a fossil fuel monopoly responds to a carbon-free substitute becoming available at some uncertain point in the future if demand is isoelastic and variable extraction costs are zero but upfront exploration investment costs have to be made. Before the breakthrough, oil reserves are depleted too rapidly; afterwards, the oil depletion rate drops and the oil price jumps up by discrete amounts. Subsidizing green R\&D to speed up the breakthrough speeds up oil extraction before the breakthrough, but more oil is left in situ as exploration investment is lower. The latter can offset the Green Paradox effect.
\end{abstract}

Keywords: regime shift, green R\&D, Green Paradox.

JEL codes: D81, H20, Q31, Q38

Submitted 22 Augustus 2017, revised 28 September 2017

\footnotetext{
*Van der Ploeg: University of Oxford, Manor Road Building, Oxford OX1 3UQ, United Kingdom (rick.vanderploeg@economics.ox.ac.uk), VU University Amsterdam, CESifo and CEPR. I gratefully acknowledge the support from the BP funded Oxford Centre for the Analysis of Resource Rich Economies. This essay has been prepared for a special issue of FinanzArchiv to mark Hans-Werner Sinn's $70^{\text {th }}$ birthday. I have benefited from the helpful comments of the editor Ronnie Schöb and an anonymous referee.
} 


\section{Introduction}

The idea that well-intended climate policy may have undesirable unintended consequences has received a lot of attention during last decade due to the seminal contribution 'Public Policies against Global Warming' by Hans-Werner Sinn (Sinn, 2008a,b,c), which has found its origin in a paper co-authored with Ngo van Long almost a quarter century earlier (Long and Sinn, 1985). This contribution, like so many other of Sinn's contributions on topics as diverse as German unification, the "bazar" economy, immigration into Europe and the financial crisis in Europe, has important policy implications and has spawned a huge literature (e.g., Gerlagh, 2011; Grafton et al., 2010; Hoel, 2010; Grafton et al., 2010, 2012; van der Ploeg and Withagen, 2012ab) which has been surveyed (e.g., van der Werff and di Maria, 2012; van der Ploeg and Withagen, 2015; Pittel et al., 2014). One way of stating Sinn's pioneering insight is that by levying a steeply rising carbon tax or subsidizing the use of renewables, owners of oil wells and gas fields anticipate capital losses on their underground reserves. They are therefore encouraged to extract and sell their oil and gas reserves more quickly rather than waiting when their operations will have become less profitable. This will exacerbate carbon emissions and global warming in the short run. This counterintuitive result has been coined the Green Paradox and is the intertemporal variant of the spatial notion of import leakage (e.g., van der Ploeg, 2016). However, if oil extraction becomes more costly as fewer reserves are left, the total amount of oil extracted from the earth is endogenous and not all oil reserves are necessarily fully exhausted. Over time, oil will become less attractive relative to the carbon-free backstop. Hence, a rising schedule for the carbon tax or a renewables subsidy makes it more attractive to keep more oil reserves in the crust of the earth and thus limit cumulative carbon emissions and peak global warming. This offsets 
and can overturn the Green Paradox, both in terms of green welfare and total welfare (van der Ploeg and Withagen, 2012a).

Our objective is to provide an alternative rationale for the Green Paradox not to hold. To make our case as stark as possible, we abstract from stock-dependent extraction costs and allow instead for initial outlays on exploration investment that determine the initial stock of oil reserves (cf., Gaudet and Laserre, 1988; Daubanes and Laserre, 2012). This also gives two margins: how quickly to extract oil and how much oil in total to extract from the earth or equivalently how much carbon emissions in total will result. We then argue that the prospect of a radical, low-cost breakthrough in the invention and bringing to the market of a carbon-free substitute (e.g., fusion energy) induces oil to be pumped up more rapidly. As a result, carbon is more quickly emitted into the atmosphere and thus global warming is exacerbated. These effects are less strong if the carbon-free backstop is a worse substitute for oil (cf., Grafton et al., 2012). At the moment the carbon-free substitute becomes available, oil use jumps down by a discrete amount and the oil price jumps up by a discrete amount unless the cost reduction of renewables and the degree of substitutability is large enough in which case the oil price jumps down. From then on, the rate of decline in the rate of oil depletion and the rate of increase in the oil price follow Hotelling paths, albeit starting from a lower level of oil reserves than if there would have been no hazard of a cheaper substitute coming to the market. This inefficiency is stronger if the risk of discovery and drop in the price of the carbon-free energy substitute are higher. Once the cheap carbon-free substitute is on the market, the rate of oil depletion follows the Hotelling rule. Uncertainty about timing of the breakthrough causes inefficiencies, not the breakthrough itself.

However, the prospect of cost-effective renewables becoming available at some random future moment implies also that exploration investment is curbed and thus that the total stock of 
available oil reserves diminishes. This inefficiency in exploration investment is a manifestation of the hold-up problem (e.g., Rogerson, 1992; Holmström and Roberts, 1998). It reduces the total of carbon emitted into the atmosphere and thus curbs global warming. Subsidizing green R\&D to bring forward the expected time of the introduction of breakthrough renewables leads to more rapid oil extraction before the breakthrough, but more oil is left in situ as exploration investment will be lower. The latter offsets and can even reverse the Green Paradox.

To highlight the inefficiencies from the eventual arrival of breakthrough carbon-free substitutes in the most striking manner, we suppose iso-elastic fossil fuel demand and zero variable resource extraction costs. This is a useful and analytically convenient benchmark, since the monopolistic resource extraction problem is efficient under these two assumptions (cf., Stiglitz, 1976).

The idea of a discrete change in demand resulting from a breakthrough technology occurring at some unknown date in the future goes back a long time (e.g., Dasgupta and Heal, 1974; Dasgupta and Stiglitz, 1981) and has recently been used to argue that innovation unsupported by carbon pricing can lead to runaway global warming worse if the carbon cycle contains strong positive feedback effects (Winter, 2014). Our contribution is to give a tractable analysis of the effects of a breakthrough in renewables technology for the path of oil extraction and exploration investment and investigate the robustness of the Green Paradox in this context.

Our model is closely related to the ones found in the literatures on potential machine failure (Kamien and Schwartz, 1971), nationalization, expropriation and confiscation risk (Long, 1975; Bohn and Deacon, 2000; Laurent-Luchetti and Santaguni, 2012; van der Ploeg, 2017), collapses of the resource stock and changes in system dynamics (regime shifts) in pollution control (e.g., Cropper, 1976; Heal, 1984; Clarke and Reed, 1994; Tsur and Zemel, 1996; Naevdal, 2006; Polasky et al., 2011; de Zeeuw and Zemel, 2012), the effects on the speed of resource extraction 
of uncertainty about the time at which a resource cartel is broken up (Benchekroun et al., 2006), and the interplay between political risk and foreign investment (e.g., Cherian and Perotti, 2001).

Section 2 presents a tractable model of oil extraction and exploration investment by a monopolistic owner of oil reserves faced with the possible arrival of breakthrough renewables. It faces a constant hazard of a breakthrough at some unknown future date. Section 3 derives the optimal oil depletion and price paths before and after the breakthrough. Section 4 characterizes the solution and gives illustrative simulations. Section 5 shows that a higher chance of a renewables breakthrough depresses exploration investment. Section 6 discusses climate policy and the Green Paradox. Section 7 summarizes results and offers suggestions for further research.

\section{The model}

We suppose that the economy needs two types of energy, viz. fossil fuel or oil for short, $O$, and renewables, $R$, which are imperfect substitutes in energy demand. Renewable energies such as solar and wind energy are getting cheaper all the time, but suffer from the problem of intermittence and thus rely on cost-effective storage (e.g., pumping water on top of hill or mountain or very efficient batteries). As long as the problem of storage is not solved, fossil fuel and renewable energy will be imperfect substitutes. Our assumption contrasts with the usual assumption of a perfect substitute (called a backstop source of energy), which is analytically convenient but less realistic.

Oil has zero extraction cost, but needs investment outlays $I$ which lead to proven initial oil reserves $S_{0}$. The price of oil is endogenous and denoted by $p$. The breakthrough occurs at time $T$ $>0$ and calendar time is denoted by $t$. Before the breakthrough $(t<T)$, renewables are infinitely 
elastically supplied at cost $\tilde{b}(t)=b$. After the breakthrough $(t \geq T)$, they are supplied at cost $\tilde{b}(t)=b-\Delta$ where $0<\Delta \leq b$. The monopolistic owner of the oil reserves chooses its level of exploration investment and extraction path to maximize the present value of its profits,

$$
\operatorname{Max}_{O, I} \mathrm{E}\left[\int_{0}^{\infty} p(t) O(t) \mathrm{e}^{-r t} d t\right]-q I
$$

subject to the oil depletion equations,

$$
\dot{S}(t)=-O(t), \forall t \geq 0, \quad S(0)=S_{0}>0, \quad \int_{0}^{\infty} O(t) d t \leq S_{0},
$$

the oil exploration investment schedule,

$$
S_{0}=\Theta(I), \quad \Theta^{\prime}>0, \Theta^{\prime \prime}<0,
$$

the oil demand schedule,

$$
O(t)=\Upsilon p(t)^{-\varepsilon} b^{\sigma}, 0 \leq t<T, \quad O(t)=\Upsilon p(t)^{-\varepsilon}(b-\Delta)^{\sigma}, \forall t \geq T,
$$

the probability that the breakthrough technology occurs in the interval ending at time $t$,

$$
\operatorname{Pr}(T \leq t)=1-\exp (-h t), \forall t \geq 0, \quad h \geq 0,
$$

where $S, q, I$ and $r$ denote the stock of oil reserves, the price of oil exploration investment, the volume of oil exploration investment and the market interest rate, respectively. The price of oil exploration investment $(q)$ and the market rate of interest $(r)$ are exogenously determined on world markets and constant over time. The concavity of $\Theta($.$) ensures decreasing returns to$ exploration investment. The own price elasticity of oil demand $(\varepsilon)$ exceeds unity, so that 
marginal oil revenue is positive. ${ }^{1}$ With the demand function (4), marginal revenue is always finite and oil reserves are fully exhausted asymptotically. Oil and renewables are supposed to be gross substitutes, so that the constant cross price elasticity of oil demand $(\sigma)$ is positive. The inverse demand function for oil is given by $p=\left(\Upsilon \tilde{b}^{\sigma} / O\right)^{1 / \varepsilon} \equiv p(O, \tilde{b})$.

The probability that the breakthrough technology has not taken place before time $t$ is $\operatorname{Pr}(T>t)=$ $\exp (-h t)$. The breakthrough will occur definitely as this probability tends to zero as time tends to infinity. The expected time for the breakthrough to occur is $\mathrm{E}[T]=1 / h$. The exponential distribution has a constant hazard rate $h$, so that the conditional probability that the breakthrough does not take place for another $t$ years given that the breakthrough has not already taken place in the first $s$ years is the same as the initial probability that the breakthrough does not take place for another $t$ years: $\operatorname{Pr}(T>s+t \mid T>s)=\operatorname{Pr}(T>t), \forall s, t \geq 0$.

\section{Optimal oil depletion paths before and after the renewables breakthrough}

Using the principle of dynamic programming, we work backward in time and first solve the deterministic problem from unknown time of the breakthrough, $T$, onwards when the cheap carbon-free substitute arrives on the market, then solve the more interesting stochastic problem of oil extraction before the substitute has arrived on the market, and finally solve for the optimal level of exploration investment. We denote the problems of oil extraction after and before the breakthrough technology with superscripts $A$ and $B$, respectively, and solve them for given $S_{0}$ in the rest of this section and characterize the outcomes in section 4 . Section 5 then solves for the optimal level of exploration investment $I$ and initial reserves $S_{0}$.

\footnotetext{
${ }^{1}$ Aggregate oil demand is relatively inelastic, but the relevant elasticity for an individual oil-producing firm is much higher as it cannot easily manipulate the price without losing market share.
} 


\section{After the breakthrough}

Marginal oil revenue must equal the oil scarcity rent, $\lambda$, which according to the Hotelling rule must rise at a rate equal to the market interest rate, $r$ :

$$
(1-1 / \varepsilon) p^{A}=\lambda, \quad \dot{\lambda} / \lambda=r
$$

It follows from (6) and the iso-elastic demand schedule (4) with zero extraction costs that the oil price and depletion paths are efficient despite the oil owner being a monopolist:

$$
\dot{p}^{A} / p^{A}=r>0, \quad \dot{O}^{A} / O^{A}=-\varepsilon r<0 .
$$

The intuition behind this rule is that the return on taking a marginal barrel out of the ground and investing it (i.e., the return on assets $r$ ) must equal the return on keeping this marginal barrel in the ground (i.e., the capital gains on underground reserves). Using (7) in (2), we solve for the optimal paths of oil depletion, oil reserves and (using (7)) the oil price after the breakthrough:

$$
\begin{gathered}
O^{A}(t)=\varepsilon r S(t)=\varepsilon r e^{-\varepsilon r(t-T)} S(T), \quad 0<S^{A}(t)=e^{-\varepsilon r(t-T)} S(T) \leq S(T)<S_{0}, \\
p^{A}(t)=e^{r(t-T)}\left[\frac{(b-\Delta)^{\sigma} \Upsilon}{\varepsilon r S(T)}\right]^{1 / \varepsilon}, \quad \forall t>T .
\end{gathered}
$$

Equations (8) imply that fossil fuel reserves are asymptotically fully depleted. They also indicate that a lower cost of the backstop $(\Delta>0)$ pushes down the oil price, especially if the backstop is a good substitute for oil (high $\sigma$ ), but does not affect the path of oil depletion rates except for depressing the final stock of oil (see outcomes before the breakthrough). Substituting (8) into (1), we get the present value of profits of the oil firm after the breakthrough technology comes to market (i.e., the value function after the regime shift):

$$
V^{A}(S(t), b-\Delta)=\left[\frac{(b-\Delta)^{\sigma} \Upsilon}{\varepsilon r}\right]^{1 / \varepsilon} S(T)^{1-1 / \varepsilon}, \quad \forall t \geq T
$$


A future breakthrough $(\Delta>0)$ reduces the cost of the substitute and thus curbs the future price of oil. As a result, the present value of oil profits is lower. Oil profits after the breakthrough are high if remaining reserves at the time of the breakthrough are high.

\section{Before the breakthrough}

The Hamilton-Jacobi-Bellman equation for the optimization problem before the breakthrough is:

$$
\operatorname{Max}_{O^{B}}\left[p\left(O^{B}, b\right) O^{B}-V_{S}^{B}(S, b, \Delta, h) O^{B}\right]-h\left[V^{B}(S, b, \Delta, h)-V^{A}(S, b-\Delta)\right]=r V^{B}(S, b, \Delta, h),
$$

where $V^{B}(S, b, \Delta, h)$ denotes the value function (i.e., the present value of profits to go excluding the cost of the initial outlay on exploration investment) before the breakthrough (see appendix for a mathematical derivation). Equation (10) states that maximum oil rents minus the expected loss in value terms of the carbon-free substitute coming to market must equal the return from investing proceeds at the market rate of interest. The maximization of oil rents in (10) requires marginal oil revenue to be set to the marginal value of in situ oil reserves:

$$
(1-1 / \varepsilon) p^{B}(t)=V_{S}^{B}(S(t), b, \Delta, h), \quad 0 \leq t<T .
$$

Using (4) and (11), we obtain the optimal oil depletion rate before the regime shift:

$$
O^{B}(t)=\Upsilon b^{\sigma}\left(\frac{V_{S}^{B}(S(t), b, \Delta, h)}{1-1 / \varepsilon}\right)^{-\varepsilon}, \quad 0 \leq t<T
$$

Upon substitution of (11) and (12) into (10), we obtain:

$$
\frac{1}{\varepsilon}\left(\frac{V_{S}^{B}(S, b, \Delta, h)}{1-1 / \varepsilon}\right)^{1-\varepsilon} \Upsilon b^{\sigma}-h\left[V^{B}(S, b, \Delta, h)-V^{A}(S, b-\Delta)\right]=r V^{B}(S, b, \Delta, h) .
$$


To solve (13), we guess the value function $V^{B}(S, b, \Delta, h)=K S^{1-1 / \varepsilon}$, substitute it with the postshift value function (9) into (13), and use the method of undetermined coefficients to solve for $K$. It then turns out that $K=K(b-\Delta, h)$ must satisfy the nonlinear equation:

$$
\frac{1}{\varepsilon} \Upsilon b^{\sigma} K^{1-\varepsilon}+h\left[\frac{(b-\Delta)^{\sigma} \Upsilon}{\varepsilon r}\right]^{1 / \varepsilon}=(r+h) K
$$

Using the resulting value function in (11) and using the oil demand function (4), we get:

$$
p^{B}(t)=K(b-\Delta, h) S(t)^{-1 / \varepsilon}, \quad O^{B}(t)=L(b, \Delta, h) S(t), \quad 0 \leq t<T .
$$

where $L(b, \Delta, h) \equiv K(b-\Delta, h)^{-\varepsilon} \Upsilon b^{\sigma}$. Solving for the time paths from (15) and (2), we obtain:

$$
p^{B}(t)=e^{L t / \varepsilon} K S_{0}^{-1 / \varepsilon}, \quad O^{B}(t)=L e^{-L t} S_{0}, \quad S^{B}(t)=e^{-L t} S_{0}, \quad 0 \leq t<T .
$$

The first part of (16) implies a distortion in the Hotelling rule, since the capital gains on keeping a barrel in the ground is now depressed by the risk of capital losses due to the breakthrough of the substitute so that the rule becomes $\left(\dot{p}^{B} / p^{B}\right)-(L-\varepsilon r) / \varepsilon=r$ with $L>\varepsilon r$ as we will see.

\section{Characterization of the solution: aggressive oil depletion}

To understand the solution more fully, we characterize the function $K=K(b-\Delta, h)$. The benchmark corresponds to a zero hazard rate. If there is no chance of a breakthrough, $h=0$, so that (14) gives $K(b-\Delta, 0)=\left(\Upsilon b^{\sigma} / \varepsilon r\right)^{1 / \varepsilon}$ and thus $O^{A}(t)=O^{B}(t)=\varepsilon r S(t), \forall t \geq 0$. Further, $L=\varepsilon r$ and thus the oil price rises at the Hotelling rate, $\dot{p}^{B} / p^{B}=r$. Conversely, if the expected time of the breakthrough is imminently small, $h \rightarrow \infty$, (14) indicates that $K \rightarrow\left[\frac{\Upsilon(b-\Delta)^{\sigma}}{\varepsilon r}\right]^{1 / \varepsilon}$ 
and $L \rightarrow \varepsilon r\left(\frac{b}{b-\Delta}\right)^{\sigma}>\varepsilon r$. The rate of oil depletion is thus faster than the Hotelling rate if the breakthrough is imminent. If the breakthrough is so radical that it leads to zero (marginal) cost of the carbon-free substitute $(b=\Delta),(14)$ gives $K(0, h)=\left[\Upsilon b^{\sigma} / \varepsilon(r+h)\right]^{1 / \varepsilon}<K(b-\Delta, 0), \forall h>0$, and thus $L(b, b, h)=\varepsilon(r+h)$. The possibility of oil being made completely obsolete thus depresses expected profits to go for any stock of oil reserves. Clearly, it also leads to more aggressive depletion of oil reserves than under efficient depletion as $\varepsilon(r+h)>\varepsilon r$.

Total differentiation of equation (14) yields:

$d K=\frac{-\frac{K}{\varepsilon h}(L-\varepsilon r) d h+\frac{h \sigma}{\varepsilon(b-\Delta)}\left[\frac{(b-\Delta)^{\sigma} \Upsilon}{\varepsilon r}\right]^{1 / \varepsilon} d(b-\Delta)}{r+h+(1-1 / \varepsilon) \Upsilon b^{\sigma} K^{-\varepsilon}}$.

Since $L=\varepsilon r$ if $h=0$ and $L>\varepsilon r$ if $h \rightarrow \infty,\left(14^{\prime}\right)$ implies $K_{h}(b-\Delta, h)<0, L_{h}(b, \Delta, h)>0, \forall h>0$.

This reflects that a higher probability of a renewables technology breakthrough reduces the expected profit to go for the oil well owner, lifts up the path for the oil depletion rate, and depresses the oil price path before the shift. It follows that $\varepsilon r<L<\varepsilon(r+h)$ for any $0<h<\infty$. Also, if $h>0, K_{b-\Delta}(b-\Delta, h)>0, \forall \Delta \in(0,1)$ from (14'). A bigger size of the climate calamity thus curbs profits to and makes oil depletion more aggressive.

Suppose that the breakthrough occurs at date $T$. We know from (16) that just before we have

$$
\begin{aligned}
& O^{B}(T-)=L e^{-L T} S_{0} \text { and } p^{B}(T-)=e^{L T / \varepsilon}\left(L S_{0}\right)^{-1 / \varepsilon} \text {. Using } S^{A}(T)=S^{B}(T)=e^{-L T} S_{0} \text { in }(8), \text { we get: } \\
& O^{A}(T+)=\varepsilon r e^{-L T} S_{0}<O^{B}(T-)=L e^{-L T} S_{0} \\
& p^{A}(T+)=\left[\frac{(b-\Delta)^{\sigma} \Upsilon}{\varepsilon r S_{0}}\right]^{1 / \varepsilon} e^{L T / \varepsilon}>p^{B}(T-)=\left(\frac{b^{\sigma} \Upsilon}{L S_{0}}\right)^{1 / \varepsilon} e^{L T / \varepsilon} \text { iff }\left(\frac{b}{b-\Delta}\right)^{\sigma}>\frac{L}{\varepsilon r}
\end{aligned}
$$


We thus arrive at the following proposition.

Proposition 1: After the breakthrough the oil depletion rate and oil reserves decline at the rate er and the oil price rises at the Hotelling rate $r$ with the corresponding time paths given by (8). Before the breakthrough the oil depletion rate and oil reserves decline too rapidly at the rate $L \equiv K(b-\Delta, h)^{-\varepsilon} \Upsilon b^{\sigma}>\varepsilon r$ and the oil price rises too rapidly at the rate $L / \varepsilon>r$ with the time paths given by (16) where $K=K(b-\Delta, h), K_{b-\Delta}>0, K_{h}<0$ solves (14). At the time of the breakthrough, there is a discrete increase fall in oil extraction. If renewables enjoy a big enough cost reduction and are a good enough substitute, the oil price falls by a discrete amount.

Anticipation of a future breakthrough in renewables technology boosts the initial oil depletion rate and depresses the initial oil price, especially if the chance of a breakthrough occurring and the expected cost reduction are high. Whilst the breakthrough technology is not on the market, the oil depletion rate falls and the oil price rises too rapid and may even cross their efficient paths. Once the breakthrough technology is on the market, the oil depletion rate jumps down and the oil price jumps down by a discrete amount if the breakthrough yields a big enough cost reduction and renewables are a good enough substitute, else the oil price jumps up by a discrete amount (see (17)). From then on oil depletion and oil prices follow Hotelling paths, but starting out from an inefficiently low level of oil reserves.

Initially, the path for oil depletion exceeds the efficient path and the oil price path is below the efficient Hotelling path, since $L>\varepsilon r$. However, if the realized date of the breakthrough is distant enough, the pre-breakthrough depletion rate can fall below and the oil price path can fall above the efficient paths. 


\subsection{Benchmark: certainty-equivalent outcome}

As benchmark we also calculate outcomes if the breakthrough in renewables technology is introduced with certainty at the expected date of the breakthrough, i.e., at time $T=1 / h$. After the breakthrough equations (8) hold. Since there cannot be a discontinuity in the price path at time

$1 / h$ and oil prices follow a Hotelling path, the initial oil price is $p(0)=e^{-r / h}\left[\frac{(b-\Delta)^{\sigma} \Upsilon}{\varepsilon r S(1 / h)}\right]^{1 / \varepsilon}$.

Equation (4) gives $O(0)=\varepsilon r e^{\varepsilon r / h}\left(\frac{b}{b-\Delta}\right)^{\sigma} S(1 / h)$ and so $O(t)=\varepsilon r e^{-\varepsilon r(t-1 / h)}\left(\frac{b}{b-\Delta}\right)^{\sigma} S(1 / h)$,

$\forall t \in[0,1 / h]$. Putting this into (2) and integrating, we get the stock of oil at the expected time of breakthrough: $S(1 / h)=\frac{S_{0}}{1+\left(e^{\varepsilon r / h}-1\right)(b /(b-\Delta))^{\sigma}} \leq e^{-\varepsilon r / h} S_{0} \leq S_{0}$. The certainty-equivalent oil price, depletion and reserves paths before and after the breakthrough can thus be calculated:

$$
S(t)=\frac{1+\left[e^{\varepsilon r(1 / h-t)}-1\right]\left(\frac{b}{b-\Delta}\right)^{\sigma}}{1+\left(e^{\varepsilon r / h}-1\right)\left(\frac{b}{b-\Delta}\right)^{\sigma}} S_{0}, t \in[0,1 / h], \quad S(t)=\frac{e^{\varepsilon r(1 / h-t)} S_{0}}{1+\left(e^{\varepsilon r / h}-1\right)\left(\frac{b}{b-\Delta}\right)^{\sigma}}, t \geq 1 / h
$$

Obviously, the path of oil depletion rates is unaffected by the cost of renewables if there is no breakthrough. However, a renewables breakthrough $(\Delta>0)$ at the known time $1 / h$ induces a lower stock of oil reserves at time $1 / h$ and thus before the breakthrough oil depletion occurs at a faster rate than after the breakthrough, especially if renewables are a good substitute for oil (high $\sigma)$. The path for oil prices satisfies the Hotelling arbitrage principle and is efficient given that the breakthrough is certain to occur at time $1 / h$. The efficient paths for oil depletion rates and oil reserves corresponding to a constant cost of renewables of either $b$ or $b-\Delta$ from time zero onwards are identical; these paths are unaffected by the breakthrough. However, the efficient oil 
price paths corresponding to cost $b$ and to $\operatorname{cost} b-\Delta$ rise at the market rate of interest $r$ and are, respectively, above and below the certainty-equivalent path of oil prices.

\subsection{Simulation of the impact of expected breakthrough in renewables technology}

To illustrate Proposition 1, fig. 1 offers some illustrative simulations of our model. We set the own price elasticity of oil to $\varepsilon=2$, the cross price elasticity of oil to $\sigma=1$ and autonomous oil demand to $\Upsilon=1$. We set the interest rate to $4 \%$ per annum, $r=0.04$. The hazard rate for the breakthrough is set to $h=0.1$, so the expected time for the breakthrough to arrive is 10 years. Hence, $0.08=\varepsilon r<L=\varepsilon(r+h)<0.28$. The cost of renewables is set to 100 before the breakthrough and to 20 after the breakthrough, so $b=100$ and $\Delta=80$. Finally, the initial stock of oil reserves is set to $S_{0}=1000$.

Fig. 1 gives various time paths for the price oil, the oil depletion rate and oil reserves. The certainty-equivalent paths(solid lines) correspond to the situation where the market believes that he breakthrough occurs at the expected date of arrival, which corresponds to the inverse of the hazard rate, $1 / h$, or ten years (see section 4.1). The time paths for the outcome where the market takes full account of uncertainty about the future date of the breakthrough correspond to different realizations of the date of the breakthrough, e.g., 10 and 25 year (short and long dashed lines).

Fig. 1 also shows two efficient time paths (dotted lines), which given zero extraction costs and isoelastic demand imply that oil prices must rise at a rate equal to the rate of interest. These correspond to the optimal outcome under a social planner with perfect foresight and where the breakthrough occurs either immediately or never. In the latter case, the price path is higher due to the higher cost of the substitute for oil. Note that the oil depletion rate and reserves are exactly the same for both these two efficient outcomes. Hotellling pricing also occurs in the certaintyequivalent outcome, but the price paths takes on an intermediate position. 


\section{Figure 1}

Impact of threat of breakthrough renewables on oil extraction and prices
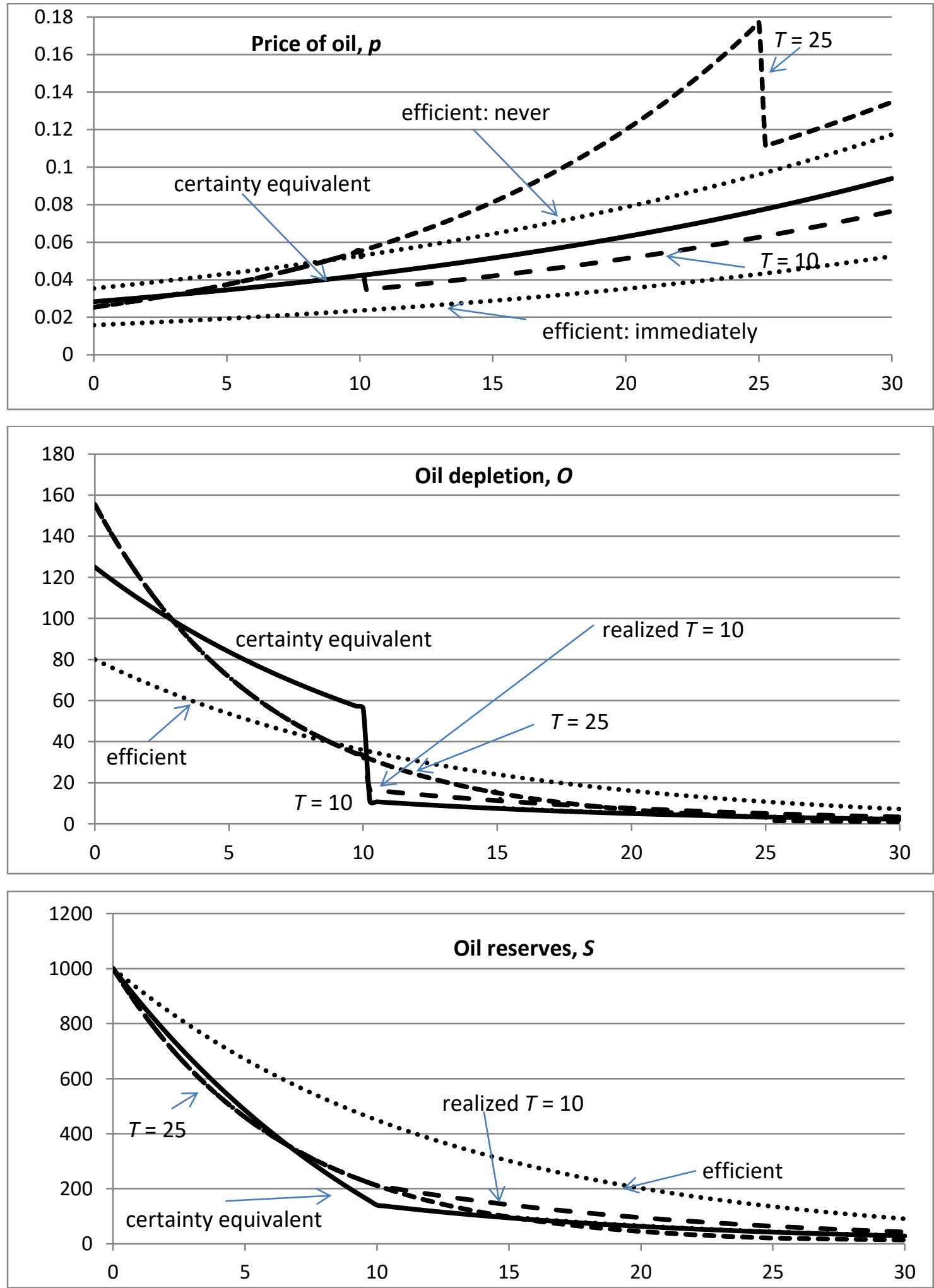
With the parameters set to our chosen values, we find that the solution to (14) is $K=0.802$ and thus that $L=0.155$. The speed of oil depletion, 0.155 , is thus almost twice as high as the speed after the breakthrough, $\varepsilon r=0.08$. Fig. 1 shows simulations with realized times of the breakthrough technology occurring at times 10 and 25 by long dashes and short dashes, respectively. We compare these with the certainty-equivalent paths (solid) and the efficient paths (dotted) when the cost of renewable energy either immediately falls or never falls. The initial oil price if there is never a breakthrough is 0.0354 and if there is an immediate breakthrough in renewables technology it is 0.0158 . From then on oil prices follow a Hotelling path in each of these two cases. As already mentioned, the paths for oil depletion rates and reserves do not depend on whether there is never or an immediate breakthrough. The certainty-equivalent path starts off with an oil price in between, 0.0283 , and then also follows a Hotelling path. Oil depletion is affected by the certainty of a breakthrough at some future date: until the breakthrough reserves are depleted at a rapid rate and therefore at a lower rate after the breakthrough.

Not knowing the date of the breakthrough also speeds up the rate of oil extraction before the breakthrough compared with the certainty-equivalent (and a fortiori the efficient) path. This means that initially oil depletion is higher and oil prices lower than in the certainty-equivalent path, but after some time as a consequence of the faster rate of oil depletion oil depletion is lower and oil prices higher than in the certainty-equivalent outcome. At the moment the breakthrough comes to market, both the rate of oil depletion and oil prices jump down and thereafter continue along their Hotelling paths, albeit from an inefficient base. If the cost reduction would have not been so substantial or the renewables would not have been such a good substitute, the oil price 
would have jumped up by a discrete moment of the breakthrough. A sufficient condition for this not to occur is that $(b /(b-\Delta))^{\sigma}>(r+h) / h=3.5$.

\subsection{Sensitivity of outcomes}

Fig. 2 plots the expected present value of oil profits at time zero, $V^{B}\left(S_{0}\right)=K(b-\Delta, h) S_{0}^{1-1 / \varepsilon}$, and the speed at which oil is extracted, $L$, against the hazard rate $h$, both for a potential cost reduction in renewables $\Delta$ of 80 and 40 . The highest feasible level of expected oil profits is 35.36 , which occurs if there is no chance of a breakthrough. The expected present value of oil profits is lower for higher hazard rates and for larger potential cost reductions in renewables.

\section{Figure 2}

Bigger risk of bigger breakthrough cuts oil profits and boosts extraction speed

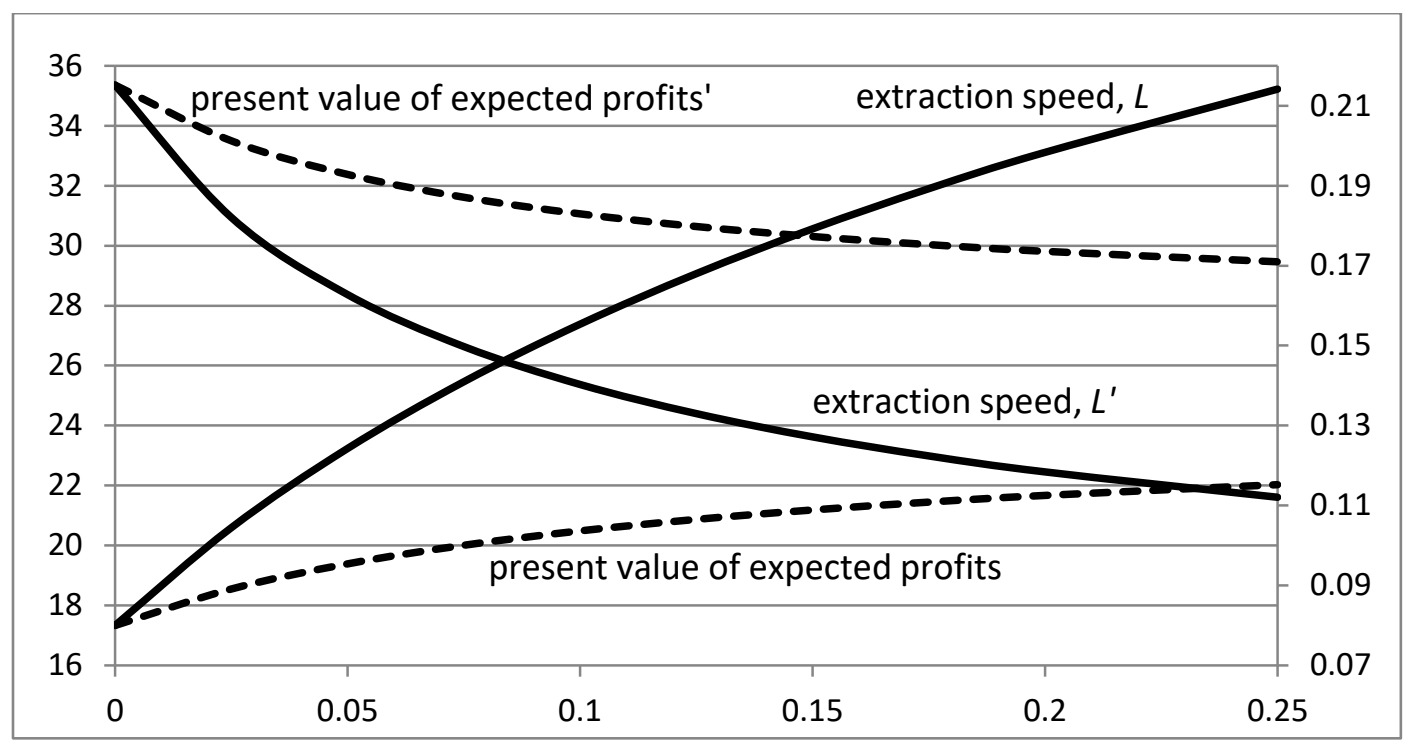

Key: Hazard rate $h$ on horizontal axis, speed of oil extraction $L$ on right vertical axis and present value of expected profits on left vertical axis. Dashed indicates $\Delta=40$ instead of 80 .

Effectively, a more serious threat of being put out of business by a revolution in breakthrough technology damages prospects for oil producers. As a result, when the threat of a breakthrough and the size of the breakthrough become more substantial, oil producers start extracting oil and more rapid rates before renewables come to market and oil prices fall. 


\section{Exploration investment and the hold-up problem}

The final stage of solving the problem stated in section 2 is to solve for the optimal level of exploration investment $I$. Using the oil exploration investment schedule (3) and the expression for the value function at time zero, $V^{B}(\Theta(I))=K(b-\Delta, h) \Theta(I)^{1-1 / \varepsilon}$, we find that this requires setting the marginal return on marginal exploration investment to its cost:

$(1-1 / \varepsilon) K(b-\Delta, h) \Theta(I)^{-1 / \varepsilon} \Theta^{\prime}(I)=q$.

Total differentiation of (19) gives $q\left[\left[\Theta^{\prime}(I) / \delta \Theta(I)\right]-\Theta^{\prime \prime}(I) / \Theta^{\prime}(I)\right] d I=q\left(K_{b} d(b-\Delta)+K_{h} d h\right) / K$ $-d q$, so that optimal exploration investment declines with its cost $q$, the breakthrough hazard $h$ and the size of the reduction in the cost of renewables after the breakthrough $\Delta$ :

$I=I(b-\Delta, h, q), \quad I_{b-\Delta}>0, I_{h}, I_{q}<0$.

If exploration investment is subsidized at the rate $\theta$, the optimality condition (19) becomes $(1-1 / \varepsilon) K(b-\Delta, h) \Theta(I)^{-1 / \varepsilon} \Theta^{\prime}(I)=q-\theta$. In the efficient outcome with never a breakthrough or a breakthrough from the outset, one has $(1-1 / \varepsilon) K(b-\Delta, 0) \Theta(I)^{-1 / \varepsilon} \Theta^{\prime}(I)=q$ with $K(b-\Delta, 0)=\left(\varepsilon r / \Upsilon b^{\sigma}\right)^{-1 / \varepsilon}$. Hence, it follows that the optimal exploration investment subsidy which corrects for the investment inefficiency increases in the breakthrough hazard and the potential cost advantage of breakthrough renewables:

$\theta=\left[\left(\varepsilon r / \Upsilon b^{\sigma}\right)^{-1 / \varepsilon}-K(b-\Delta, h)\right](1-1 / \varepsilon) \Theta(I)^{-1 / \varepsilon} \Theta^{\prime}(I) \equiv \theta(\Delta, h)>0, \quad \theta_{\Delta}>0, \theta_{h}>0$.

Equations (20) and (21) give rise to the following proposition. 
Proposition 2: The inefficiencies induced by the uncertain timing of a breakthrough in renewables are exacerbated by a drop in exploration investment, especially if the risk of a better carbon-free substitute and the potential cost reduction are higher. These inefficiencies can be eliminated by subsidizing exploration investment at the rate (21).

This proposition is an illustration of the hold-up problem (e.g., Rogerson, 1992; Holmström and Roberts, 1998). One way to overcome this is vertical integration, which may be feasible if the government can nationalize the oil firm. There may also be contractual solutions. Here an appropriate exploration investment subsidy gets rid of the inefficiency. As oil producers are typically in different jurisdictions to oil users, such a subsidy is unlikely to be implemented.

Finally, note that if the market knows with certainty that a breakthrough technology will arrive at a given known future date, the optimal exploration investment subsidy is zero as there is no point of a subsidy if the breakthrough will definitely happen at a future date and cannot be brought forward by a subsidy.

\section{Climate policy and the Green Paradox}

It is easy to see that, again under the assumptions of iso-elastic demand and zero oil extraction costs, a constant ad valorem carbon tax which is equivalent to a fall in oil demand (lower $\Upsilon$ ) or a constant subsidy to renewables use (lower $b$ throughout) do not affect the paths of oil extraction and oil reserves. A rising path of ad valorem carbon taxes or a constant specific carbon tax will affect the rate of oil depletion, but we will abstract from these for it is difficult to muster political support for these policies. Policy makers find the carrot easier than the stick, so they focus at subsidizing green $R \& D$ instead of taxing carbon emissions. Subsidizing green $R \& D$ is meant to 
bring forward the introduction of carbon-free substitutes for fossil fuel, so we specify $h=H(\varpi), H^{\prime}>0, H^{\prime \prime}<0$, where $\varpi$ is the subsidy for green R\&D. The subsidy thus increases the hazard rate $h$ and cuts the expected time of the breakthrough, $1 / h$. We see from (20) and Proposition 2 (abstracting from exploration investment subsidies) that subsidizing green R\&D makes oil more obsolete and thus depresses exploration investment $I$ and thus curbs the total amount of recoverable oil reserves, $S_{0}$, and the total amount of carbon that can be emitted into the atmosphere. We also know from Proposition 1 that a higher hazard rate slows down the speed of oil extraction and thus the speed at which carbon is emitted into the atmosphere (see also fig. 2). Subsidizing green R\&D to speed up the development of carbon-free substitutes thus leads to a Green Paradox in the short run. However, as initial exploration investment and initial fossil fuel reserves are curbed, the total amount of carbon that can be emitted into the atmosphere is curbed, the Green Paradox can in principle be reversed in the long run.

To see this a little more precisely, define the present value of global warming damages by:

$$
G \equiv \int_{0}^{\infty} D\left(E_{0}+\Theta(I(b-\Delta, H(\varpi)))-S(t)\right) e^{-\rho t} d t, \quad D^{\prime}>0, D^{\prime \prime}>0,
$$

where $E_{0}>0$ denotes the initial stock of carbon in the atmosphere, $\rho>0$ the social rate of discount and $D($.$) denotes the damages from atmospheric carbon (as a proxy for global$ warming). This formulation supposes, for simplicity, that all carbon that is emitted into the atmosphere stays there forever. Total carbon in the biosphere is thus $E_{0}+S_{0}$ and $E \equiv E_{0}+S_{0}-\mathrm{S}$ thereof is in the atmosphere and contributes to global warming. Global warming damages depend on total carbon emissions and are described by the convex function $D(E)=D\left(E_{0}+\Theta(I)-S\right)$. As oil in measured in Giga tons of carbon, we then have: 
$\frac{\partial G}{\partial \varpi}=\underbrace{-\int_{0}^{\infty} \frac{\partial S(t)}{\partial h} H^{\prime}(\varpi) D^{\prime}(E(t)) e^{-\rho t} d t}_{+}+\underbrace{\Theta^{\prime} I_{h} H^{\prime} \tau}_{-}$,

where $\tau \equiv \int_{0}^{\infty} D^{\prime}(E(t)) e^{-\rho t} d t>0$ defines the social cost of carbon (the present value of marginal damages caused by emitting one extra ton of carbon today). The first term on the right-hand side of (23) indicates that a green R\&D subsidy speeds up oil extraction and thus exacerbates damages, which is the usual (weak) Green Paradox, and the second term shows that the subsidy discourages exploration of oil fields and thus limits the total amount of carbon emitted into the atmosphere. If the latter effect dominates, the Green Paradox is reversed and a subsidy for investment in green breakthrough $R \& D$ exacerbates damages from global warming. More generally, this occurs if the price elasticity of supply of fossil fuel reserves is high and that of demand for fossil fuel is low (van der Ploeg, 2016). In the original analysis of the Green Paradox initial reserves were given and supply completely inelastic in which case a renewable energy subsidy also produces a Green Paradox and higher damages from global warming.

\section{Conclusion}

We have used a tractable model of a resource-owning monopolist with iso-elastic demand and zero variable oil extraction costs to gain more insights into the Green Paradox. The anticipation of the arrival of a carbon-free substitute at an uncertain moment of time in the future induces oil well owners to pump oil more quickly and to push down the oil price. Since this leads to more rapid emissions of the given amount carbon in the crust of the earth, global warming is exacerbated which is the (weak) Green Paradox. As soon as the carbon-free substitute has arrived, the oil depletion rate jumps down. If the new carbon-free fuel is not a very good 
substitute and the cost reduction is not too substantial, the oil price jumps up by a discrete amount at that moment. If the breakthrough is substantial enough and a good enough substitute for oil, the oil price jumps down. From then on the oil depletion rate declines at the Hotelling rate, albeit starting out from a lower level of reserves than would be the case if there was no anticipation of renewables being introduced, and the oil price rises at the market rate of interest. Interestingly, if the carbon-free substitute was introduced from the outset with certainty, oil extraction would be unaffected.

An uncertain introduction date for the carbon-free substitute depresses oil exploration investment and thus more oil is left in the crust of the earth which is due to hold-up problem. Hence, the total amount of carbon emitted into the atmosphere is reduced, albeit that what is emitted is emitted more rapidly. This can easily overturn the Green Paradox in the sense that the present discounted value of global warming damages can increase. The exploration investment inefficiency can be corrected for with an appropriate subsidy, which increases in the chance of the breakthrough occurring and the cost reduction arising from the breakthrough technology.

The consequences of cheaper renewables are thus not necessarily as bleak as suggested by proponents of the Green Paradox. Future work will benefit from a better grasp of regime shifts, whether they relate to arrival of carbon-free substitutes or potential tipping points and climate disasters. ${ }^{2}$ It is also of interest to put the breakthrough approach in a strategic setting. ${ }^{3}$

\footnotetext{
${ }^{2}$ If positive feedback effects in the carbon cycle are strong enough, runaway global warming may result (Winter, 2014). However, this will lead to an irreversible doomsday scenario.

${ }^{3}$ Hoel (1978) and Gerlagh and Liski (2011) analyze the strategic pricing policies of an oil producer faced with a substitute coming to market. Jaakkola (2012) analyzes in more detail the strategic dynamic interactions with oil importers developing substitutes. Jaakkola and van der Ploeg (2017) perform primarily a numerical analysis of an international dynamic game in carbon pricing, on the one hand, and investments in breakthrough technology, on the other hand.
} 


\section{References}

Bechekroun, H., Gaudet, G. and Long, N. Van (2006), Temporary Natural Resource Cartels, Journal of Environmental Economics and Management 52, 663-674.

Bohn, H. and Deacon, R. (2000), Ownership Risk, Investment, and the Use of Natural Resources, American Economic Review 90, 526-49.

Cherian, J.A. and Perotti, E. (2001), Option Pricing and Foreign Investment under Political Risk, Journal of International Economics 55, 359-377.

Clarke, H.R. and Reed, W.J. (1994), Consumption/Pollution Tradeoffs in an Environment Vulnerable to Pollution-Related Catastrophic Collapse, Journal of Economic Dynamics and Control 18, 991-1010.

Cropper, M.L. (1976), Regulating Activities with Catastrophic Environmental Effects, Journal of Environmental Economics and Management 3, 1-15.

Dasgupta, P. and Heal, G.M. (1974), The Optimal Depletion of Exhaustible Resources, Review of Economic Studies 41, 3-28.

Dasgupta, P. and Stiglitz, J. (1981), Resource Depletion under Technological Uncertainty, Econometrica 49, 85-104.

Daubanes, J. and Laserre, P. (2012), Non-Renewable Resource Supply: Substitution Effect, Compensation Effect and All That, mimeo., ETH, Zurich.

Gaudet, G. and Laserre, P. (1988), On Comparing Monopoly and Competition in Exhaustible Resource Exploitation, Journal of Environmental Economics and Management 15, 412418.

Gerlagh, R. (2011), Too Much Oil, CESifo Economic Studies 57, 79-102.

Gerlagh, R. and Liski, M. (2011), Strategic Resource Dependence, Journal of Economic Theory 146, 699-727.

Grafton, R.Q., Kompas, T. and Long, N. Van (2010), Biofuels and the Green Paradox, Working Paper 2960, CESifo, Munich.

Grafton, R.Q., Kompas, T. and Long, N. Van (2012), Substitution between Bio-Fuels and Fossil Fuels: Is there a Green Paradox?, Journal of Environmental Economics and Management $64,328-341$. 
Heal, G.M. (1984), Interactions between Economy and Climate: A Framework for Policy Design Uncertainty, 151-168 in V. Kerry Smith and A. White (eds.), Advances in Applied Micro-Economics, Vol. 3, JAI Press, Greenwich, Connecticut.

Hoel, M. (1978), Resource Extraction, Substitute Production, and Monopoly, Journal of Economic Theory 19, 28-37.

Hoel, M. (2010), Is there a Green Paradox?, Working Paper 3168, CESifo, Munich.

Holmström. B. and Roberts, J. (1998), The Boundaries of the Firm Revisited, Journal of Economic Perspectives, 12, 73-94.

Hotelling, H. (1931), The Economics of Exhaustible Resources, Journal of Political Economy 39, 137-175.

Jaakkola, N. (2012), Strategic Oil Supply and Development of Substitutes, mimeo., University of Oxford.

Jaakkola, N. and Ploeg, F. van der (2017), Non-Cooperative and Cooperative Climate Policies with Anticipated Breakthrough Technology, OxCarre Research Paper 190, University of Oxford.

Kamien, M.I. and Schwartz, N.L. (1971), Optimal Maintenance and Sale Age for a Machine subject to Failure, Management Science 17, 495-504.

Laurent-Luchetti, J. and Santuguni, M. (2012), Ownership Risk and the Use of Common-Pool Natural Resources, Journal of Environmental Economics and Management 63, 242-259.

Long, N. Van (1975), Resource Extraction under the Uncertainty about possible Nationalization, Journal of Economic Theory 10, 42-53.

Long, N. Van and Sinn, H.W. (1985), Surprise Price Shifts, Tax Changes and the Supply Behavior of Resource Extracting Firms, Australian Economic Papers 24, 278-289.

Naevdal, E. (2006). Dynamic Optimization in the Presence of Threshold Effects when the Location of the Threshold is Uncertain - With an Application to a possible Disintegration of the Western Antarctic Ice Sheet, Journal of Economic Dynamics and Control 30, 1131-1158.

Pittel, K., Ploeg, F. van der and Withagen, C. (2014), Climate Policy and Non-Renewable Resources - The Green Paradox and Beyond, MIT Press, Cambridge, Mass. 
Ploeg F. van der (2016), Second-Best Carbon Taxation in the Global Economy: The Green Paradox and Carbon Leakage Revisited, Journal of Environmental Economics and Management, 78, 85-105.

Ploeg, F. van der (2017), Political Economy of Dynamic Resource Wars, Journal of Environmental Economics and Management, forthcoming.

Ploeg, F. van der and Withagen, C. (2012a), Is there Really a Green Paradox?, Journal of Environmental Economics and Management 64, 342-363.

Ploeg, F. van der and Withagen, C. (2012b), Too Much Coal, Too Little Oil, Journal of Public Economics 96, 62-77.

Ploeg, F. van der and Withagen,C. (2015). Global Warming and the Green Paradox: A Review of Adverse Effects of Climate Policy, Review of Environmental Economics and Policy 9, 285-303.

Polaski, S., Zeeuw, A.J. de and F. Wagener, F. (2011), Optimal Management with Potential Regime shifts, Journal of Environmental Economics and Management 62, 229-240.

Rogerson, W. (1992), Contractual Solutions to the Hold-Up Problem, Review of Economic Studies 59, 774-794.

Sinn, H.W. (2008a), Public Policies Against Global Warming, International Tax and Public Finance 15, 360-394.

Sinn, H.W. (2008b), Das Grüne Paradoxon: Warum mann das Angebot bei der Klimapolitik nicht vergessen darf, Perspectiven der Wirtschaftspolitik 9, 109-142.

Sinn, H.W. (2008c), Das Grüne Paradoxon - Plädoyer für eine Illusionsfrei Klimapolitik, Econ, Berlin.

Stiglitz, J.E. (1976), Monopoly and the Rate of Extraction of Exhaustible Resources, American Economic Review 66, 655-661.

Tsur, Y. and Zemel, A. (1996), Accounting for Global Warming Risks: Resource Management under Event Uncertainty, Journal of Economic Dynamics and Control 20, 1289-1305.

Werf, E. van der and Maria, C. Di (2012), Imperfect Environmental Policy and Polluting Emissions: The Green Paradox and Beyond, International Review of Environmental and Resource Economics 6, 153-194.

Winter, R. A. (2014), Innovation and the Dynamics of Global Warming, Journal of Environmental Economics and Management 68, 124-140. 
Zeeuw, A.J. de and Zemel, A. (2012), Regime Shifts and Uncertainty in Pollution Control, Journal of Economic Dynamics and Control 36, 939-950.

\section{Appendix: Derivation of the HJB equation (10)}

Since the probability of a regime shift in an infinitesimally small time period $\Delta t$ is $h \Delta t$, the Principle of Optimality from the perspective of time zero can be written as follows:

$$
\begin{aligned}
e^{-r t} V^{B}(S(t))=\operatorname{Max}_{O^{B}} & {\left[\int_{t}^{t+\Delta t} e^{-r s} p\left(O^{B}(s)\right) O^{B}(s) d s+(1-h \Delta t) e^{-r(t+\Delta t)} V^{B}(S(t+\Delta t))\right.} \\
+ & \left.h \Delta t e^{-r(t+\Delta t)} V^{A}(S(t+\Delta t))\right]
\end{aligned}
$$

(suppressing the arguments $\mathrm{b}, \Delta$ and $\mathrm{h}$ in the value function $V^{B}($.$) ). Multiplying both sides by e^{r t}$, rearranging and dividing by $\Delta t$, we rewrite (A1) as:

$$
\begin{gathered}
\operatorname{Max}_{O^{B}}\left[\frac{\int_{t}^{t+\Delta t} e^{-r(s-t)} p\left(O^{B}(s)\right) O^{B}(s) d s}{\Delta t}-h e^{-r \Delta t} V^{B}(S(t+\Delta t))+h e^{-r \Delta t} V^{A}(S(t+\Delta t))\right. \\
\left.\frac{\left(e^{-r \Delta t}-1\right) V^{B}(S(t+\Delta t))}{\Delta t}+\frac{V^{B}(S(t+\Delta t))-V^{B}(S(t))}{\Delta t}\right]=0 .
\end{gathered}
$$

Evaluating the integral in (A2) for infinitesimally small $\Delta t$ and taking the limit as $\Delta t \rightarrow 0$ whilst using l'Hôpital's Rule for $\lim _{\Delta t \rightarrow 0} \frac{\exp (-r \Delta t)-1}{\Delta t}=-r$, we get:

$$
\underset{O^{B}}{\operatorname{Max}}\left[p\left(O^{B}(t)\right) O^{B}(t)-\dot{V}^{B}(S(t))-h V^{B}(S(t))+h V^{A}(S(t))-r V^{B}(S(t))\right]=0
$$

Substituting $\dot{V}^{B}=V_{S}^{B} \dot{S}$ and using (2), rearranging and dropping the time index, we get (10). 